\title{
$b$-hadron production at ATLAS and CMS experiments
}

\author{
Eduard De La Cruz Burelo*† \\ Department of Physics \\ CINVESTAV IPN, \\ Mexico City, Mexico \\ E-mail: eduardefis.cinvestav.mx
}

We report on a selected number of studies performed by the ATLAS and the CMS collaborations on $b$-hadron production. Both experiments have a rich program on $b$-hadron physics exploiting the large cross section of $b$-hadrons at the high energies of the LHC.

38th International Conference on High Energy Physics

3-10 August 2016

Chicago, USA

\footnotetext{
* Speaker.

${ }^{\dagger}$ The author wants to aknowledge the financial support of CONACYT (FOINS-296-2016) and the Mexican HEP Network (Red FAE) to attend to the conference.
} 


\section{Introduction}

The production fo $b$-hadrons have been an important experimental tool to understand QCD, and for this reason several experiments at CERN and Fermilab had included these as an essential part of their physics programs. With the Run 2 of the LHC, the $b$-hadron production at energies never reached before provides a new input to test theoretical calculations, and the large statistics opens a new window to search for new and exotic decay channels.

In this contribution a summary of selected studies performed by the ATLAS [1] and the CMS [2] experiments is reported. Both experiments have a rich program on $b$-hadron physics and they have produced a large number of new results that were not included due to lack of time and space. However several other contributions in this conference include some of the new results not covered here.

\section{Quarkonium cross sections at $13 \mathrm{TeV}$ and non-prompt fraction}

At ATLAS and CMS, the $b$-hadron production studies are based mainly on the reconstruction of $J / \psi \rightarrow \mu^{+} \mu^{-}$decays, since their represent excellent tools to calibrate the response of detectors owing to their relatively large branching fraction into di-lepton pairs, allowing simple and reliable candidate selection and reconstruction methods to be used. In addition, those $J / \psi$ coming from a $b$-hadron decay are in average separated from the main collision point. Both experiments have studied [3,4] the production of heavy quarkonium (the bound state of a heavy quark and antiquark) and measured their production cross section and the non-prompt fraction (the fraction not coming from the main interaction point). Figures 1 and 2 show the measurements performed on the quarkonium sector in data at $13 \mathrm{TeV}$.
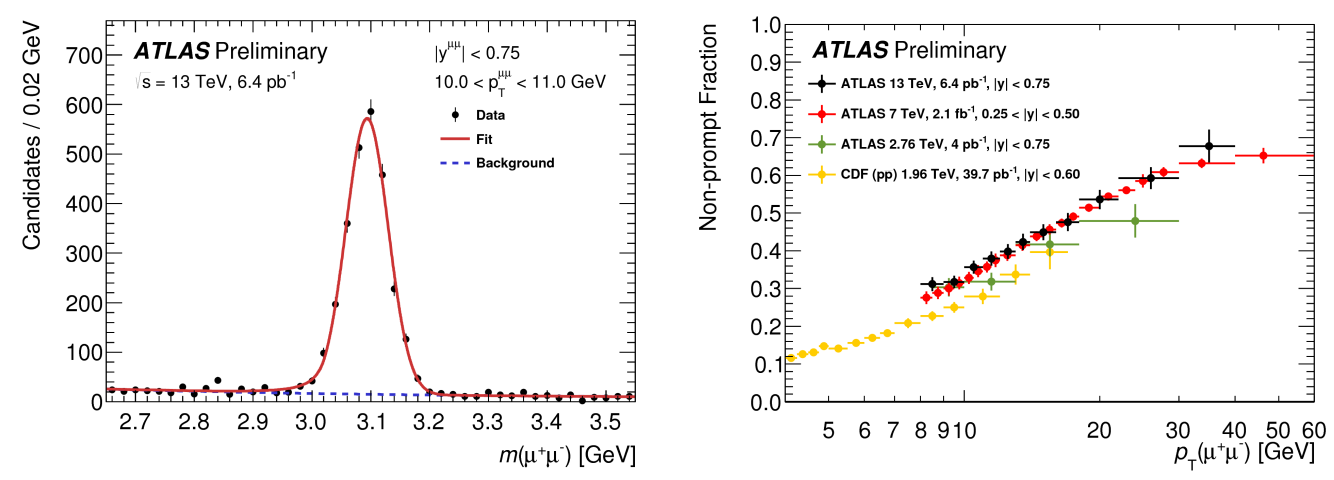

Figure 1: Left: Invariant mass distribution of $J / \psi \rightarrow \mu^{+} \mu^{-}$decays in the ATLAS data. Right: Non-prompt fraction of $J / \psi \rightarrow \mu^{+} \mu^{-}$decays.

\section{3. $B^{+}$production at $13 \mathrm{TeV}$}

The $B^{+}$meson is the b-hadron most copiously produced of all $b$-hadrons, and as such the one used for first measurements or as a detector performance tool. In the ATLAS experiment, the $B^{+}$ 

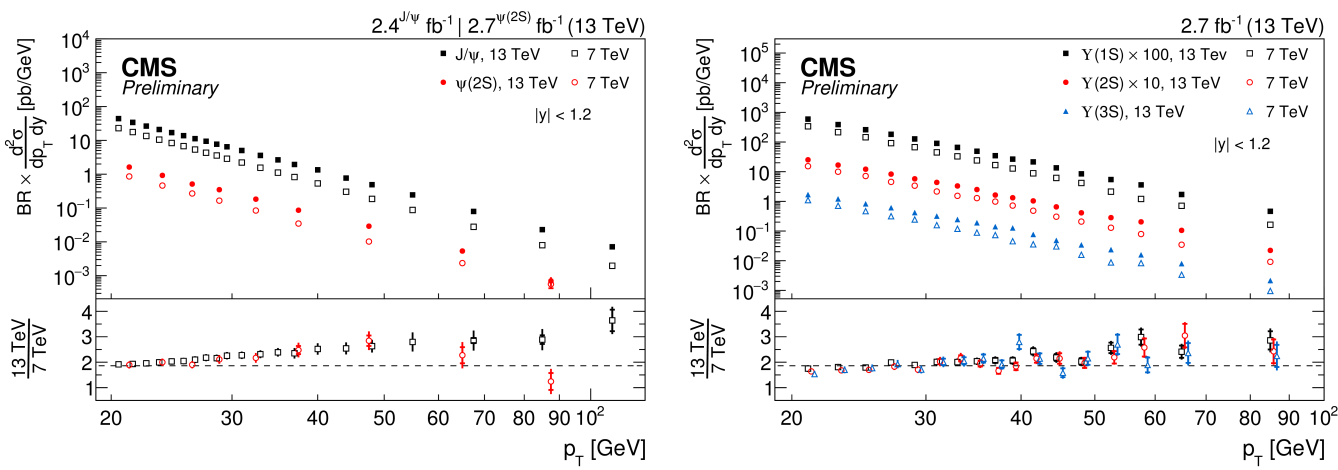

Figure 2: Differential cross sections times branching ratios for $7 \mathrm{TeV}[5,6]$ and $13 \mathrm{TeV} \mathrm{CMS}$ data, for $\psi(n S)$ (left) and $\Upsilon(n S)$ (right), for $|y|<1.2$, assuming isotropic dimuon decays. The inner error bars represent the statistical uncertainty while the total errors show the statistical and systematic uncertainties. The uncertainty on the luminosity measurement is not included.

was reconstructed in the decay channel $B^{+} \rightarrow J / \psi K^{+}$, with $J / \psi \rightarrow \mu^{+} \mu^{-}$in $3.2 \mathrm{fb}^{-1}$ of data to test the momentum calibration of the tracker detector [7]. Figure 3 shows the mass distribution of the $B^{+}$in the ATLAS data and also the mass measurement performed for this meson by ATLAS.
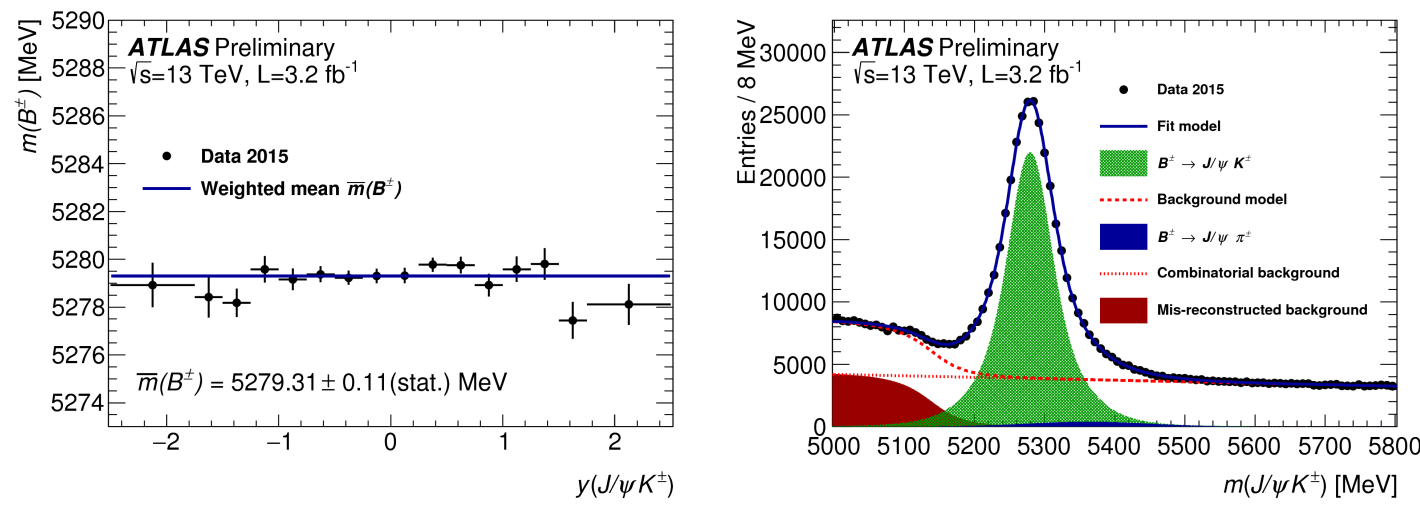

Figure 3: Left: Mass of the $B^{+}$in bins of the rapidity $y$. Right: Invariant mass distribution of the $B^{+} \rightarrow$ $J / \psi K^{+}$for the entire rapidity sample.

The CMS collaboration reconstructed the $B^{+}$in the same decay channel but using a data sample of $50.8 \mathrm{pb}^{-1}$ [8]. In these data, CMS measured the differential cross section for the $B^{+}$and compared it with predictions, finding a reasonable agreement with FONNL calculations and with the results obtained with the PYTHIA [9] event generator.

\section{Observation of $B^{+} \rightarrow \psi(2 S) \phi K^{+}$in the CMS data}

Recently several experiments have reported the observation of structures in the $J / \psi \phi$ mass spectrum from $B^{+} \rightarrow J / \psi \phi K^{+}$decays $[10,11,12,13,14,15,16]$. A natural extension of these 

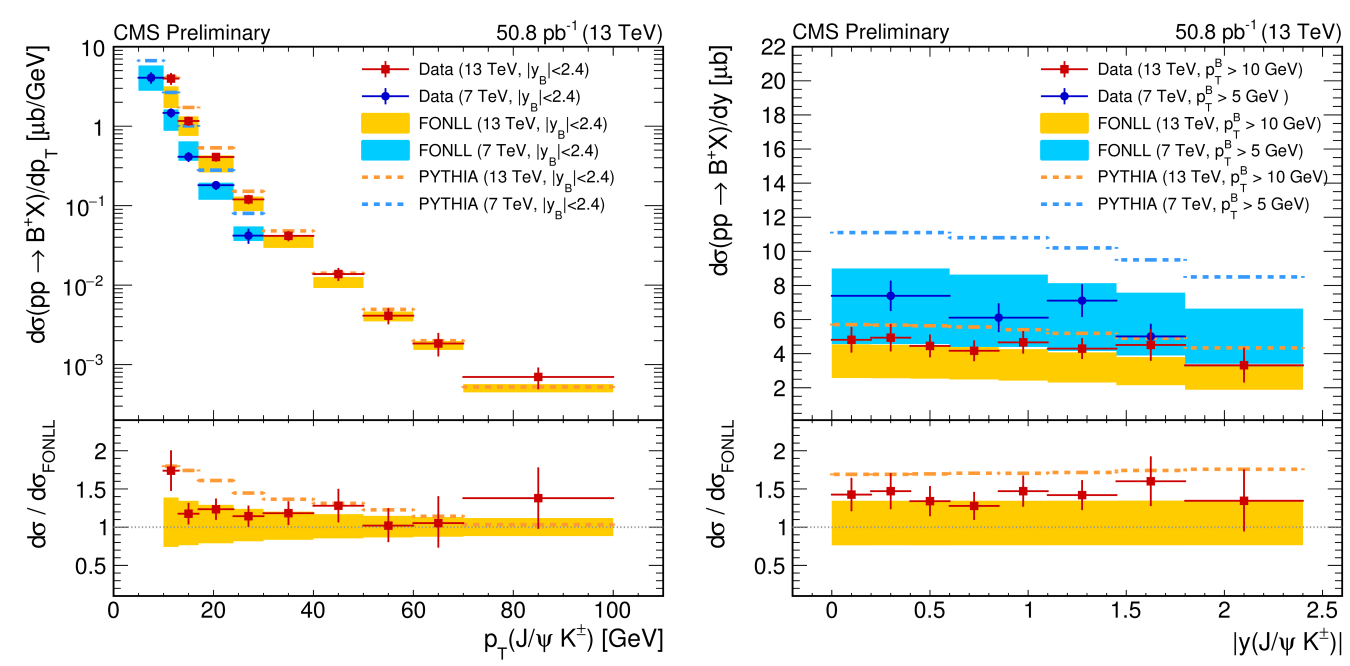

Figure 4: Differential cross sections $d \sigma / d p_{T}$ for $|y|<2.4$ (left) and $d \sigma / d y$ for $10<p_{T}<100 \mathrm{GeV}$ (right), for $B^{+}$production in $p p$ collisions at $\mathrm{s}=13 \mathrm{TeV}$.

searches is to study the mass spectra of $\psi(2 S) \phi K^{+}$and $\psi(2 S) \phi$ decays. The CMS experiment performed such studies and as part of them CMS reported the first observation of $B^{+} \rightarrow \psi(2 S) \phi K^{+}$ decays [17], with $\psi(2 S) \rightarrow \mu^{+} \mu^{-}$and $\phi \rightarrow K^{+} K^{-}$. The CMS collaboration measured the corresponding branching fraction using $19.6 \mathrm{fb}^{-1}$ of data collected in pp collisions at $8 \mathrm{TeV}$. The branching fraction was measured using as a reference channel the decay $B^{+} \rightarrow \psi(2 S) K^{+}$. Figure 5 shows the invariant mass distribution for the $B^{+}$reconstructed in the CMS data.
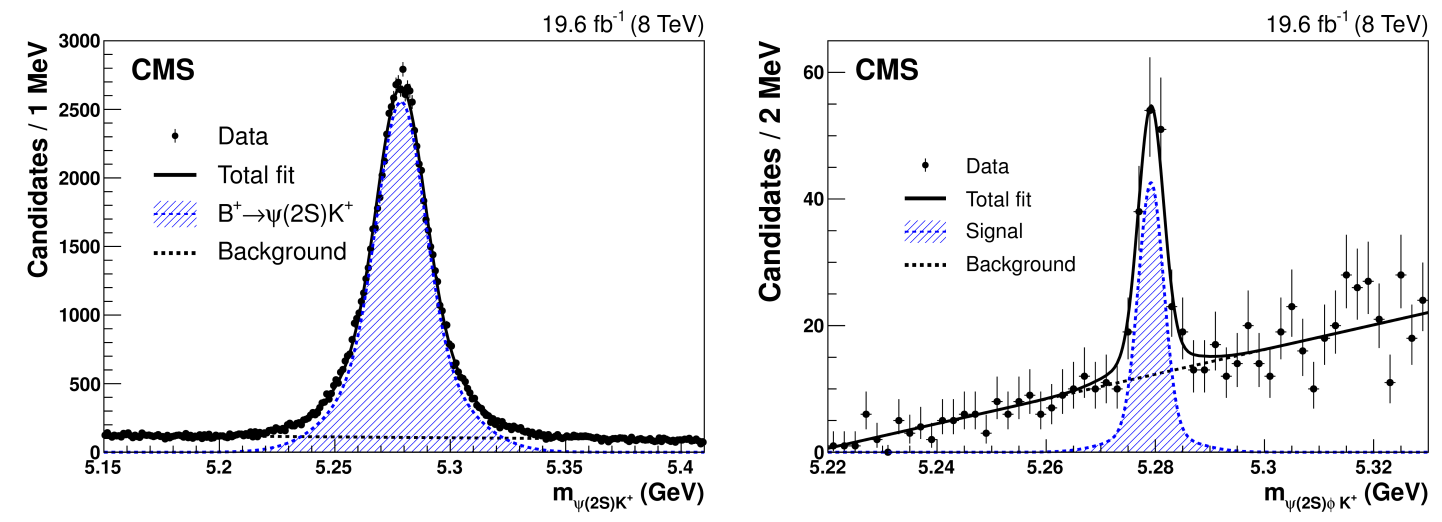

Figure 5: Invariant mass distribution of $B^{+} \rightarrow \psi(2 S) K^{+}$(left) and $B^{+} \rightarrow \psi(2 S) \phi K^{+}$(right).

The branching fraction $B r\left(B^{+} \rightarrow \psi(2 S) \phi K^{+}\right)$was determined to be $4.0 \pm 0.4($ stat $) \pm 0.6($ syst $) \pm$ $0.2(B r)) \times 10^{-6}$ where the third uncertainty reflects the imprecision in the value of $B r\left(B^{+} \rightarrow\right.$ $\left.\psi(2 S) K^{+}\right)$. 


\section{Search for the $X(5568)$ in the CMS data}

The evidence of a new $\mathrm{B}_{\mathrm{s}}^{0} \pi^{ \pm}$state presented by the DØ Collaboration [18] awakened a lot of interest within the community that studies exotic hadrons [19] and triggered this search at several hadron collider experiments, including CMS [20] and LHCb [21].

The CMS search for resonance-like structures in the $\mathrm{B}_{\mathrm{s}}^{0} \pi^{ \pm}$invariant mass spectrum was performed using an integrated luminosity of $19.7 \mathrm{fb}^{-1}$ of $p p$ collisions at $\sqrt{s}=8 \mathrm{TeV}$. The $\mathrm{B}_{\mathrm{s}}^{0}$ candidates are reconstructed in the decay chain $\mathrm{B}_{\mathrm{s}}^{0} \rightarrow \mathrm{J} / \psi \phi, \mathrm{J} / \psi \rightarrow \mu^{+} \mu^{-}, \phi \rightarrow \mathrm{K}^{+} \mathrm{K}^{-}$. The $\mathrm{B}_{\mathrm{s}}^{0} \pi^{ \pm}$ invariant mass distributions do not show any unexpected structures for different kinematic requirements imposed to the $\pi^{ \pm}, \mathrm{B}_{\mathrm{s}}^{0}$ and $\mathrm{B}_{\mathrm{s}}^{0} \pi^{ \pm}$candidates. An upper limit on the relative production of $X(5568)$ and $B_{s}$ multiplied by the branching fraction of the decay $X(5568) \rightarrow B_{s} \pi^{ \pm}$is estimated to be $3.9 \%$ at $95 \% \mathrm{CL}$ in the most conservative case.
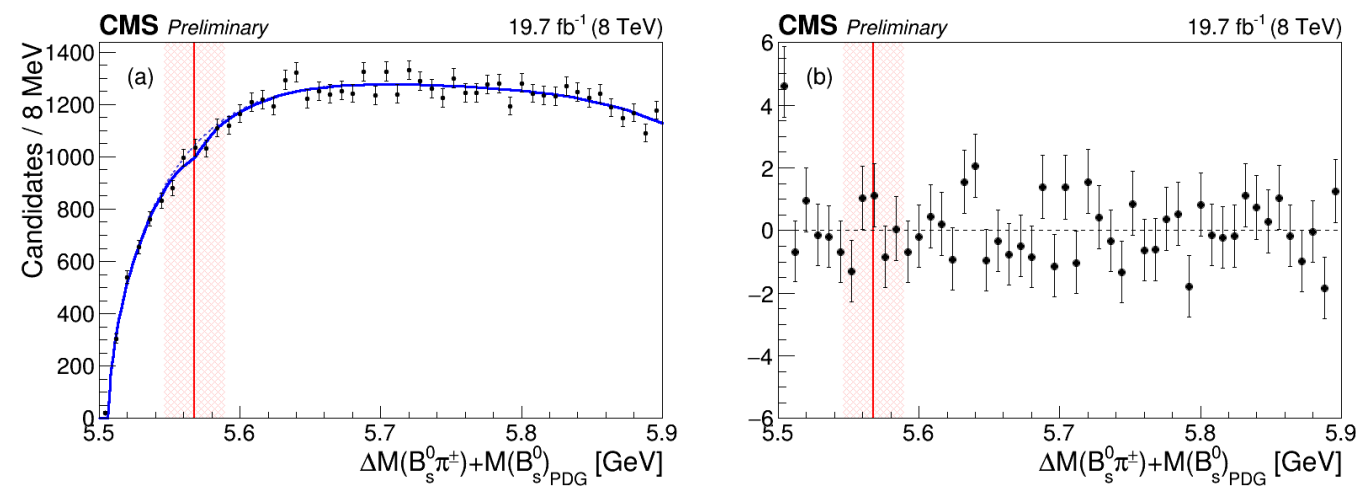

Figure 6: (a) Mass distribution of events in the $B_{s}$ signal region (black points with error bars) with fit results superimposed (blue line). (b) The pull distribution for (a). The (red) vertical band indicates the region of the $\mathrm{D}$ observation around the mass of the claimed state.

\section{Summary}

Both experiments, ATLAS and CMS, have a rich program on $b$-hadron production, exploiting their large cross section at the high energies of the LHC. This has allows both experiments to measured production cross sections, to observe new decays, and to search for new exotic decays. ATLAS and CMS have oriented their trigger on dimuons, suitable for studies on rare decays of $b$ hadrons, so new results on that line will come soon, in addition to properties and other observations

\section{References}

[1] G. Aad et al. The ATLAS Experiment at the CERN Large Hadron Collider. JINST, 3:S08003, 2008.

[2] S. Chatrchyan et al. The CMS experiment at the CERN LHC. JINST, 3:S08004, 2008.

[3] The ATLAS collaboration. Measurement of the differential non-prompt $J / \psi$ production fraction in $\sqrt{s}=13 \mathrm{TeV}$ pp collisions at the ATLAS experiment. 2015. 
[4] CMS Collaboration. Quarkonium production cross sections in pp collisions at $\sqrt{s}=13 \mathrm{TeV}$. 2016.

[5] Vardan Khachatryan et al. Measurement of $J / \psi$ and $\psi(2 S)$ Prompt Double-Differential Cross Sections in pp Collisions at $\sqrt{s}=7$ TeV. Phys. Rev. Lett., 114(19):191802, 2015.

[6] Vardan Khachatryan et al. Measurements of the $\Upsilon(1 S), \Upsilon(2 S)$, and $\Upsilon(3 S)$ differential cross sections in pp collisions at $\sqrt{s}=7 \mathrm{TeV}$. Phys. Lett., B749:14-34, 2015.

[7] The ATLAS collaboration. $B^{ \pm}$mass reconstruction in $B^{ \pm} \rightarrow J / \psi K^{ \pm}$decay at ATLAS at 13 $\mathrm{TeV} p p$ collisions at the LHC. 2015.

[8] CMS Collaboration. Measurement of the $\mathrm{B}^{+}$hadron production cross section in pp collisions at $13 \mathrm{TeV} .2015$.

[9] Torbjorn Sjostrand, Stephen Mrenna, and Peter Z. Skands. A Brief Introduction to PYTHIA 8.1. Comput. Phys. Commun., 178:852-867, 2008.

[10] T. Aaltonen et al. Evidence for a Narrow Near-Threshold Structure in the $J / \psi \phi$ Mass Spectrum in $B^{+} \rightarrow J / \psi \phi K^{+}$Decays. Phys. Rev. Lett., 102:242002, 2009.

[11] R Aaij et al. Search for the $X(4140)$ state in $B^{+} \rightarrow J / \psi \phi K^{+}$decays. Phys. Rev., D85:091103, 2012.

[12] Victor Mukhamedovich Abazov et al. Search for the $\mathrm{X}(4140)$ state in $B^{+} \rightarrow J / \psi \phi K^{+}$decays with the D0 detector. Phys. Rev., D89(1):012004, 2014.

[13] Serguei Chatrchyan et al. Observation of a peaking structure in the $J / \psi \phi$ mass spectrum from $B^{ \pm} \rightarrow J / \psi \phi K^{ \pm}$decays. Phys. Lett., B734:261-281, 2014.

[14] J. P. Lees et al. Study of $B^{ \pm, 0} \rightarrow J / \psi K^{+} K^{-} K^{ \pm, 0}$ and search for $B^{0} \rightarrow J / \psi \phi$ at BABAR. Phys. Rev., D91(1):012003, 2015.

[15] Roel Aaij et al. Observation of $J / \psi \phi$ structures consistent with exotic states from amplitude analysis of $B^{+} \rightarrow J / \psi \phi K^{+}$decays. 2016.

[16] Roel Aaij et al. Amplitude analysis of $B^{+} \rightarrow J / \psi \phi K^{+}$decays. 2016.

[17] Vardan Khachatryan et al. Observation of the decay $\mathrm{B}^{+} \rightarrow \psi(2 \mathrm{~S}) \phi(1020) \mathrm{K}^{+}$in pp collisions at $\sqrt{s}=8 \mathrm{TeV}$. Submitted to: Phys. Lett. B, 2016.

[18] V. M. Abazov et al. Evidence for a $B_{s}^{0} \pi^{ \pm}$state. Phys. Rev. Lett., 117(2):022003, 2016.

[19] Feng-Kun Guo, Ulf-G. Mei $\beta$ ner, and Bing-Song Zou. How the X(5568) challenges our understanding of QCD. Commun. Theor. Phys., 65(5):593-595, 2016.

[20] CMS Collaboration. Search for the X(5568) state in $B_{s} \pi^{ \pm}$decays. 2016.

[21] The LHCb Collaboration. Search for structure in the $B_{s}^{0} \pi^{ \pm}$invariant mass spectrum. LHCbCONF-2016-004. 